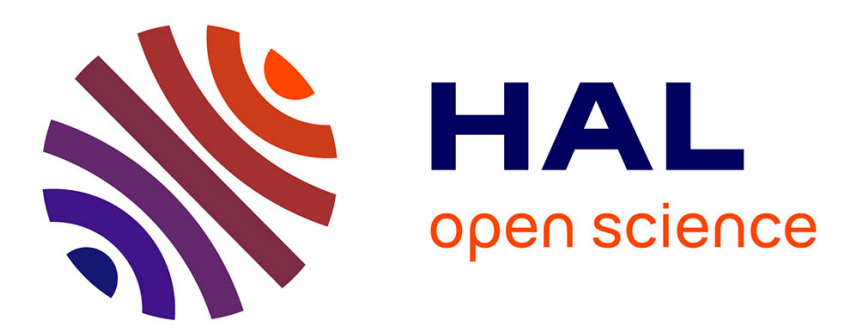

\title{
Co-design of aircraft vertical tail and control laws with distributed electric propulsion and flight envelop constraints
}

\author{
Eric Nguyen Van, Doll Carsten, Daniel Alazard, Philippe Pastor
}

\section{To cite this version:}

Eric Nguyen Van, Doll Carsten, Daniel Alazard, Philippe Pastor. Co-design of aircraft vertical tail and control laws with distributed electric propulsion and flight envelop constraints. CEAS Aeronautical Journal, 2021, 12 (1), pp.101-113. 10.1007/s13272-020-00481-8 . hal-03130562

\section{HAL Id: hal-03130562 https://hal.science/hal-03130562}

Submitted on 3 Feb 2021

HAL is a multi-disciplinary open access archive for the deposit and dissemination of scientific research documents, whether they are published or not. The documents may come from teaching and research institutions in France or abroad, or from public or private research centers.
L'archive ouverte pluridisciplinaire HAL, est destinée au dépôt et à la diffusion de documents scientifiques de niveau recherche, publiés ou non, émanant des établissements d'enseignement et de recherche français ou étrangers, des laboratoires publics ou privés. 


\title{
Co-design of aircraft vertical tail and control laws with distributed electric propulsion and flight envelop constraints
}

\author{
Eric Nguyen Van · Daniel Alazard · Carsten Döll · Philippe Pastor
}

Received: date / Accepted: date

\begin{abstract}
A sequential co-design framework has been developed in a previous study [20] to design an aircraft using active differential thrust. Differential thrust is used instead of a rudder to generate the yawing moment. The objective is to dimension in parallel the vertical tail surface area, the electric motor bandwidth and control laws while maintaining imposed handling qualities. This paper focuses on the development of a single step co-design taking into account handling qualities, flight envelop requirements and motor saturation. Additional and compatible optimisation constraints are found based on a sensitivity analysis. It reveals the importance of electric motor bandwidth with respect to aircraft natural stability. The direct co-design leads to an optimised trade off between vertical tail and electric motor bandwidth.
\end{abstract}

Keywords Distributed electric propulsion · differential thrust · vertical tail reduction · aircraft handling qualities · co-design $\cdot H_{\infty}$ control design

\section{Introduction}

Distributed Electric Propulsion (DEP) opens new aircraft design possibilities made possible by a judicious propulsion air-frame integration. Advantages associated with this technology are favorable aero-propulsive interactions leading to an increase of the low speed performances or lower drag [15] [5], better propulsive efficiency [17], relaxation of

The authors would like to thank Airbus through the CEDAR chair and ONERA for supporting this work.

Eric Nguyen Van

ISAE SUPAERO

10 Avenue Edouard Belin

BP 54032 - 31055 Toulouse CEDEX 4

E-mail: eric.nguyen-van@isae.fr design requirements [3], increase of lateral control authority [12] and lower environmental impact [26]. Among these disciplines, this work focuses on the possibilities offered by increased lateral control authority using differential thrust. Additionally, the characteristic rapid response time of electric motors allows a more active use of the propulsion systems to control the lateral dynamics. The increased lateral control, rapid actuators and higher redundancy represent a change of paradigm for the design of the vertical tail and rudder.

In previous studies, it was found that a large directional flight envelop could be achieved using differential thrust, a reduced vertical tail without rudder, in normal and degraded flight conditions [22], [21]. This study was further advanced

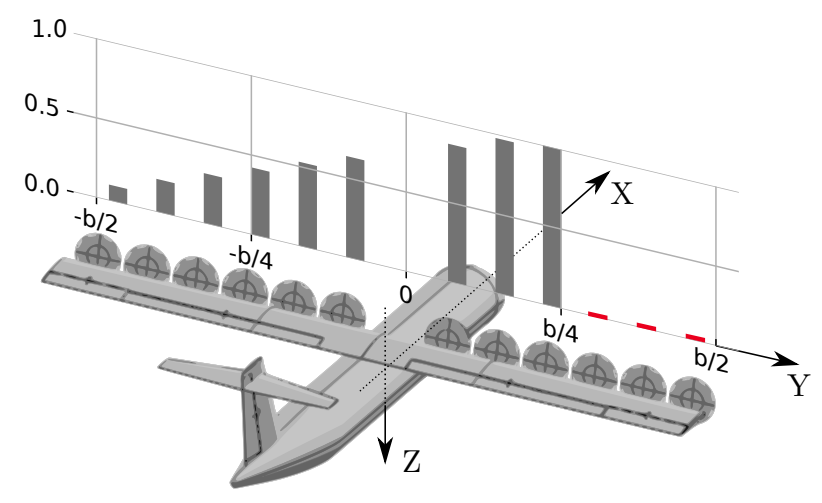

Fig. 1: The aircraft and its distributed propulsion considered in this study with three electric motor failures.

to dynamic consideration with the idea of assessing a lateral control without rudder, relying only on the differential use of the propulsion systems. 
A co-design approach based on multi-objective optimisation techniques similar to [7], was presented in [20] as a methodology to dimension in parallel the vertical tail surface area, control laws and electric motor bandwidth while complying with flight handling requirements. A sequential optimisation methodology was employed to gain knowledge of the system. The drawbacks of this method are manual tuning of parameters and a trade-off between vertical tail size, electric motor bandwidth and control effort that remains the designer's choice. To obtain an optimal trade-off in a single step, the optimisation must be constrained with additional requirements. Additional constraints representative of a prescribed directional flight envelop are proposed with consideration of the available control power. To help defining these constraints such that they lead to a feasible design, a sensitivity analysis is conducted by studying the impact of aircraft vertical tail surface area and electric motor bandwidth on the control effort.

This paper is divided in two parts, section 2 describes the aircraft, propulsion, aerodynamic and flight dynamics models and the control structure. Section 3 details the codesign methodology, sensitivity analysis and the new constraints formulations.

\section{Methodology}

\subsection{Aircraft and propulsion model}

The aircraft considered in this study is an ATR72, a subsonic regional transport aircraft in the 70 pax class. The aircraft main characteristics are detailed in Table 1. The inertia terms are based on the report [19]. The DEP version of the ATR72 considered here is propelled by twelve electric motors of equal power, distributed uniformly at the wing leading edge. These motors are equipped with propellers. Each motor is seen as an actuator that can be controlled individually. This feature mitigates the effect of one or many motor failures as thrust can be redistributed so as to avoid an asymmetric thrust as illustrated in Figure 1. The geometry and mass properties of the ATR72 are re-used for the study of the DEP version of the aircraft.

In practice, the propulsion forces, $\mathbf{F}_{T}$ and moments, $\mathbf{M}_{T}$ are computed as follow:

$$
\begin{aligned}
& \mathbf{F}_{T}=\sum_{i=1}^{N}\left(\begin{array}{c}
T_{i} \cos \left(i_{p, i}\right) \\
0 \\
T_{i} \sin \left(i_{p, i}\right)
\end{array}\right), \\
& \mathbf{M}_{T}=\sum_{i=1}^{N}\left(\begin{array}{l}
x_{i} \\
y_{i} \\
z_{i}
\end{array}\right) \times\left(\begin{array}{c}
T_{i} \cos \left(i_{p, i}\right) \\
0 \\
T_{i} \sin \left(i_{p, i}\right)
\end{array}\right),
\end{aligned}
$$

Table 1: ATR 72 general details [2], [16]

\begin{tabular}{l|c} 
Variables & Value \\
\hline Wingspan & $27 \mathrm{~m}$ \\
Wing surface area & $61 \mathrm{~m}^{2}$ \\
VT surface area & $12 \mathrm{~m}^{2}$ \\
Motor level arm & $4.1 \mathrm{~m}$ \\
Mass & $21500 \mathrm{Kg}$ \\
$I_{x}$ & $289873 \mathrm{Kg} \cdot \mathrm{m}^{2}$ \\
$I_{y}$ & $298442 \mathrm{Kg} \cdot \mathrm{m}^{2}$ \\
$I_{z}$ & $573579 \mathrm{Kg} \cdot \mathrm{m}^{2}$ \\
Continuous mechanical power & $4000 \mathrm{KW}$ \\
Stall velocity $V_{\mathrm{sr}}$ & $52 \mathrm{~m} / \mathrm{s}$ \\
Propeller efficiency, $\mu_{p}$ & 0.7
\end{tabular}

where $T_{i}$ and $x_{i}, y_{i}, z_{i}$ are respectively the thrust force and the position of the $i^{\text {th }}$ motor with respect to the center of gravity. Motors are labeled such that the outer left (negative $Y$ ) is the $1^{\text {st }}$ one and the outer right (positive $Y$ ) is the $12^{\text {th }}$ one. $i_{p, i}$ is the motor's tilt angle with respect to $X$-axis.

A thrust model considering electric motors independent from air density is used (see [25]). It is further assumed that the total electrical power on-board the aircraft is fully available at all time. The model for computing $T_{i}$ is defined by equation 3 .

$T_{i}=\frac{P_{E}}{N} V^{-1} \eta_{m} \eta_{p} \delta_{x, i}$

with $P_{E}$ being the total electrical power available for propulsion on the aircraft, $N$ being the total number of motors, $\eta_{m}$ and $\eta_{p}$ respectively the motor and propeller efficiency (both considered constant), $\delta_{x, i}$ the throttle command of the $\mathrm{i}^{\text {th }}$ motor.

\subsection{Aircraft aerodynamic model}

In order to compute the general aircraft aerodynamic model, the aircraft is modelled in OpenVSP [13]. A Vortex Lattice Method (VLM), VSPAero, is used to compute the aerodynamic derivatives of a geometry omitting motors and the vertical tail.

The change in aircraft aerodynamic coefficients as function of vertical tail size is conveniently made by a semiempirical model: VeDSC [6]. This model addresses specifically regional subsonic transport aircraft and takes into account interactions between the vertical tail and other aircraft components, namely the wing, fuselage and horizontal tail.

The model is used as follow: each lateral aerodynamic coefficient $\left\{C_{Y}, C_{l}, C_{n}\right\}$, can be decoupled in three contributions as illustrated for $C_{Y}$ in equation 4 .

$C_{\mathrm{Y}}=C_{\mathrm{Y}, F}+C_{\mathrm{Y}, W}+C_{\mathrm{Y}, v}$, 
where subscripts $F, W, v$ refer respectively to fuselage, wing and vertical tail. In this formulation, the effects of the fuselage $C_{\mathrm{Y}, F}$ and the wing $C_{\mathrm{Y}, W}$, are determined with the rest of the coefficients in VLM computation. $C_{Y, v}$, on the contrary is calculated based on the vertical tail lift slope coefficient $a_{v}$ and vertical tail position on the aircraft. The same applies to $C_{l}$ and $C_{n}$ with models such as proposed by Etkin [11]. VeDSC gives a direct method to estimate $a_{v}$ as a function of vertical tail geometry, corrected for the contribution of the fuselage, wing and horizontal tail as illustrated in equation 5 .

$a_{v}=K_{F} K_{W} K_{H} C_{L, v_{\beta}}$,

with $C_{L, v_{\beta}}$ the lift slope of a swept wing determined using Diederich formula for swept wing [8]. $K_{F}, K_{W}$ and $K_{H}$ are corrective coefficients accounting respectively for the fuselage, the wing and the horizontal tail geometry and position [22], [23]. The advantage of this method lies in the fact that it is a direct method, allowing to modify the vertical tail geometry and update the aircraft aerodynamic coefficients rapidly. Batch evaluation of aircraft stability with diverse vertical tail design and size is rendered fast, an advantage for co-design.

Aero-propulsive interactions between propellers and the wing can produce important local lift increase. As a consequence of local lift increase, when differential thrust is used, a non-negligible induced rolling moment can be produced [21], [9]. Among the geometrical parameters that influence propeller-wing interactions, is found the position of the motors on the wing and the maximum propeller disk loading, mainly set by the propeller diameter and motor power [27]. These parameters are not considered as decision variables for the co-design. This is justified by the fact that a distributed propulsion architecture is most likely only loosely influenced by the size of the vertical tail. Other considerations such as wing bending stress, favourable propeller/wing interactions, or available propulsive equipment, to cite only a few, are believed to be, all together, most influencing for the distributed architecture.

In consequence, no control can be exerted during codesign to minimize or maximize the effects of interaction: it is a side effect. The focus being the development of the co-design methodology, aero-propulsive interactions will be ignored for the rest of the study. Therefore, thrust is identical for each motor, linear with the power level $\delta_{x, i}$ (no loss of momentum due to aero-propulsive effect) and rolling moment induced by differential thrust is ignored. A dedicated study will take place to take these side effects into consideration.

\subsection{Aircraft flight dynamic model}

The baseline mathematical model is the flight dynamics equations expressed in the aerodynamic reference frame:

$m\left(\begin{array}{c}\dot{V} \\ \dot{\beta} V \\ \dot{\alpha} V \cos \beta\end{array}\right)+\mathbf{T}_{a b}\left(\begin{array}{c}p \\ q \\ r\end{array}\right) \times\left(\begin{array}{l}V \\ 0 \\ 0\end{array}\right)=m \mathbf{g}+\mathbf{F}_{A}+\mathbf{F}_{T}$,

$\mathbf{I}\left(\begin{array}{c}\dot{p} \\ \dot{q} \\ \dot{r}\end{array}\right)+\left(\begin{array}{c}p \\ q \\ r\end{array}\right) \times \mathbf{I}\left(\begin{array}{c}p \\ q \\ r\end{array}\right)=\mathbf{M}_{A}+\mathbf{M}_{T}$,

with $m$ the mass, $\mathbf{g}$ the gravity vector, $\mathbf{I}$ the inertia matrix, $\mathbf{F}_{A}, \mathbf{F}_{T}$ and $\mathbf{M}_{A}, \mathbf{M}_{T}$ are respectively the force and torque vectors due to aerodynamics and propeller thrust. $\mathbf{T}_{a b}$ is the rotation matrix between the body carried frame and the aerodynamic frame. $V, \alpha, \beta$, being the airspeed, the angle of attack and the side slip angle, and $p, q$ and $r$, the roll, pitch and yaw rates. The complementary kinematic equations for the Euler angles: bank $\phi$, pitch $\theta$ and heading $\psi$ are:

$$
\left(\begin{array}{c}
\dot{\phi} \\
\dot{\theta} \\
\dot{\psi}
\end{array}\right)=\left(\begin{array}{ccc}
1 & \sin \phi \tan \theta & \cos \phi \tan \theta \\
0 & \cos \phi & -\sin \phi \\
0 & \frac{\sin \phi}{\cos \theta} & \frac{\cos \phi}{\cos \theta}
\end{array}\right)\left(\begin{array}{l}
p \\
q \\
r
\end{array}\right) .
$$

Two additional parameters are necessary to find pseudo-equilibriums: the flight path angle $\gamma$ and the turn rate $\Omega$. For steady turns, $\Omega$ is equal to the rate of change of heading and using the last line of equation 8 :

$$
\begin{aligned}
\sin \gamma & =\cos \alpha \cos \beta \sin \theta-\sin \beta \sin \phi \cos \theta \\
& -\sin \alpha \cos \beta \cos \phi \cos \theta, \\
\Omega & =(q \sin \phi+r \cos \phi) \frac{1}{\cos \theta} .
\end{aligned}
$$

Leaving aside the last row of system (8), the system to solve consists in equations (6), (7), (8), (9) and (10) representing a set of $N_{e}=10$ equations. The state vector is $\mathbf{x}=$ $[V, \alpha, \beta, p, q, r, \phi, \theta]^{T}$ and counts $n_{x}=8$ variables. The directional control being entirely made with differential thrust, the input vector corresponding to control surfaces is composed of ailerons and elevator deflection: $\mathbf{u}=\left[\delta_{a}, \delta_{e}\right]^{T}$ with $n_{u}=2$, to which are added the motor throttle command $\delta_{x, i}$. The total input vector is: $\mathbf{u}=\left[\delta_{a}, \delta_{e}, \delta_{x, 1}, \ldots, \delta_{x, N}\right]^{T}$, with $n_{u}=2+12=14$. Finally, $n_{p}=2$ additional parameters $\gamma$ and $\Omega$ are counted.

\subsection{System linearisation and Linear Fractional Representation}

The math model described in section 2.3 is used with the propulsion and aerodynamic model described in section 2.1 
and 2.2 to extract a set of linear systems describing the aircraft flight dynamics for different vertical tail sizes. The procedure is only recalled here and the interested reader is referred to [20] for the detailed methodology.

- A trim position if found at a desired velocity and altitude while the climb angle, side slip and turning rate are maintained at zero. As the system is over-determined, an optimisation algorithm based on Sequential Least SQuare Programming algorithm (see [18]) is used to this end.

- Linear systems are obtained by first order Taylor expansion from the trim position. The system is finally reorganised into longitudinal and lateral dynamics as in equations

$$
\dot{\mathbf{x}}_{L}=\mathbf{A}_{L} \mathbf{x}_{L}+\mathbf{B}_{L} \mathbf{u}_{L}
$$$$
\dot{\mathbf{x}}_{D}=\mathbf{A}_{D} \mathbf{x}_{D}+\mathbf{B}_{D} \mathbf{u}_{D}
$$

with:

$$
\begin{aligned}
\mathbf{x}_{L} & =[\widetilde{V}, \widetilde{\gamma}, \widetilde{\alpha}, \widetilde{q}]^{T}, \\
\mathbf{u}_{L} & =\left[\widetilde{\delta}_{e}, \widetilde{\delta}_{x, 1}, \ldots, \widetilde{\delta}_{x, 12}\right]^{T}, \\
\mathbf{x}_{D} & =[\widetilde{\beta}, \widetilde{p}, \widetilde{r}, \widetilde{\phi}]^{T}, \\
\mathbf{u}_{D} & =\left[\widetilde{\delta}_{a}, \widetilde{\delta}_{x, 1}, \ldots, \widetilde{\delta}_{x, 12}\right]^{T},
\end{aligned}
$$

where $\sim$ means the variation of the corresponding variable with respect to its trim value.

- A set of lateral linearised models are computed for vertical tail varying from $0.1 S_{\mathrm{v}, 0}$ to $1.2 S_{\mathrm{v}, 0}$ with steps of $0.1 S_{\mathrm{v}, 0}$. The collection of models is then put into an Linear Fractional Representation (LFR), $\mathbf{M}-\boldsymbol{\Delta}$ form. This form allows the representation of the lateral aircraft dynamics as a function of a single varying parameter, here the vertical tail size ratio $\delta_{v}=\frac{S_{v}}{S_{v_{0}}}$. This parameter is treated as a simple gain in a block diagram scheme. The toolbox APRICOT and the Matlab function lsapprox are used to this end. See [24] for more details about the APRICOT toolbox.

The set of linearised lateral systems further permits to investigate the impact of the variation of the vertical tail surface area on the aircraft lateral dynamics. The evolution of the Dutch-Roll poles is presented in Figure 2.

This result is obtained for the flight condition : $[V, \beta, \gamma, \Omega]=$ $\left[1.3 V_{\mathrm{sr}}, 0^{\circ}, 0^{\circ}, 0^{\circ} / \mathrm{s}\right]$ which was selected as particularly sizing for differential thrust control. At $1.3 V_{\mathrm{sr}}$, the pilot requires a wide flight envelop while the control efficiency of propulsion is inversely proportional to the velocity (see equation 3 and [21]).

As the vertical tail reduces, the side slip oscillation gradually becomes unstable and changes to an aperiodic behaviour for values lower than $\delta_{v}=0.2$ with two unstable real poles. In parallel, as the vertical tail becomes smaller the control effort will be increased to maintain handling qualities. An

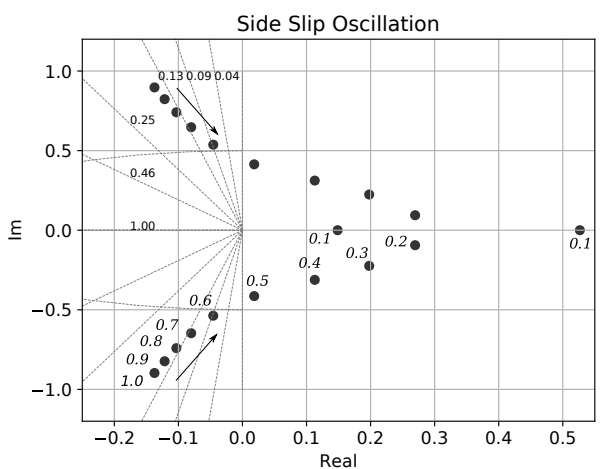

Fig. 2: Evolution of the eigenvalues of the side slip oscillation of the reference aircraft with the reduction of the vertical tail surface area, from $S_{\mathrm{v}} / S_{\mathrm{v}_{0}}=1$ to $S_{\mathrm{v}} / S_{\mathrm{v}_{0}}=0.1$. The number in italic next to an eigenvalue indicates the corresponding vertical tail size ratio $\delta_{v}=S_{\mathrm{v}} / S_{\mathrm{v}_{0}}$. The regular number next to a dashed line corresponds to the damping value.

optimal trade-off between vertical tail surface area and handling qualities at the cost of a reasonable control effort should exist and the objective is to define a systematic methodology to deduce this trade-off.

\subsection{Control architecture}

The longitudinal/lateral flight control laws is depicted in Figure 3 and involve:

- a static feed-forward gain $\mathbf{H}_{L}(2 \times 2)$, resp. $\mathbf{H}_{D}(2 \times 2)$ and a static feedback gain $\mathbf{K}_{L}(2 \times 2)$, resp. $\mathbf{K}_{D}(2 \times 4)$, for the longitudinal, resp. lateral, flight control,

- a longitudinal inner loop to control the short period mode through the gains $K_{\alpha}$ and $K_{q}$,

- the symmetrical and differential thrust allocation matrices $\mathbf{L}_{L}(6 \times 1)$ and $\mathbf{L}_{D}(6 \times 1)$, respectively.

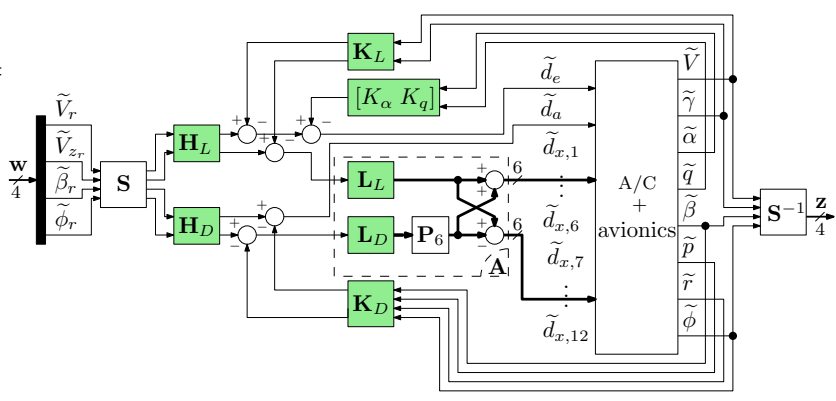

Fig. 3: The longitudinal/lateral closed-loop control blockdiagram. 
This set of gains (in green boxes in Figure 3) are the control decision variables $\mathcal{K}$ to be tuned by the optimization process:

$\mathcal{K}=\left\{\mathbf{H}_{L}, \mathbf{H}_{D}, \mathbf{K}_{L}, \mathbf{K}_{D}, K_{\alpha}, K_{q}, \mathbf{L}_{L}, \mathbf{L}_{D}\right\}$.

The whole allocation matrix $\mathbf{A}$, for the 12 throttle commands, reads:

$\mathbf{A}_{12 \times 2}=\left[\begin{array}{cc}\mathbf{1}_{6} & \mathbf{1}_{6} \\ \mathbf{P}_{6} & -\mathbf{P}_{6}\end{array}\right]\left[\begin{array}{cc}\mathbf{L}_{L} & \mathbf{0}_{6 \times 1} \\ \mathbf{0}_{6 \times 1} & \mathbf{L}_{D}\end{array}\right]$,

where the permutation matrix $\mathbf{P}_{n}$ is defined by:

$\mathbf{P}_{n}(i, j)=\left\{\begin{array}{l}1 \text { if: } i+j=n+1, \\ 0 \text { otherwise. }\end{array}\right.$

In addition, $\mathbf{S}=\operatorname{diag}\left(\left[1, \frac{1}{V}, \frac{\pi}{180}, \frac{\pi}{180}\right]\right)$ is an input shaping gain to take into account the reference input on vertical velocity $V_{z} \approx V \gamma$ since requirements specify decoupling between $V$ and $V_{z}$ (see for example [10]), the side slip and bank angle reference inputs $\widetilde{\beta}_{r}$ and $\widetilde{\phi}_{r}$ are expressed in degrees. Thus, a unitary step on each of the 4 components of the reference input $\mathbf{w}$ is expected to create roughly the same thrust magnitude.

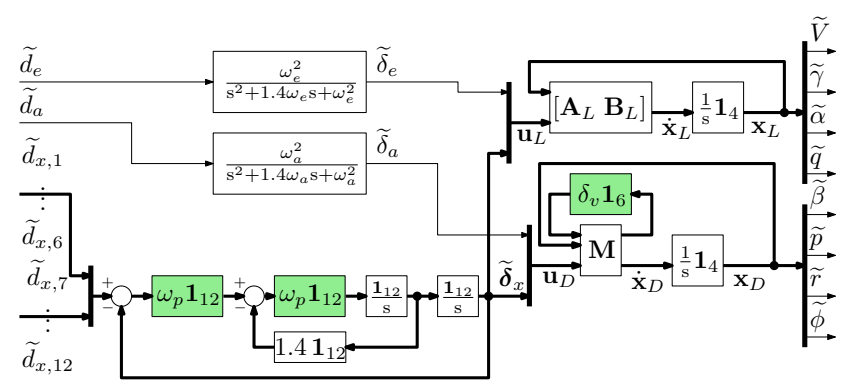

Fig. 4: The A/C + avionics block-diagram.

The aircraft (A/C) and avionics block of Figure 3 is detailed in Figure 4. It consists of the longitudinal and lateral models presented in the previous section completed by the avionics model between the required control signals $\widetilde{d}_{e}, \widetilde{d}_{a}$, and $\widetilde{d}_{x, i}(i=1, \cdots 12)$ computed by the control law and the really applied actuations $\widetilde{\delta}_{e}, \widetilde{\delta}_{a}$ and $\widetilde{d}_{x, i}$. This avionics block considers a second order model on each of the 14 actuators with a damping ratio of 0.7 and a cut-off frequency of $\omega_{e}, \omega_{a}$ and $\omega_{p}$ on the elevator, the ailerons and the 12 propeller motors, respectively. Thus, 2 additional decision variables are added for the optimization process: the vertical tail surface ratio $\delta_{v}$ (6 occurrences) and the propeller motor bandwidth $\omega_{p}$ (24 occurrences).

Actuator saturation in position and in thrust is considered for time-domain simulation and through constraints on the RMS gain for $H_{\infty}$ control design.

\section{Co-design}

The handling qualities are expressed through frequency-domain templates $\mathbf{S}_{o, \text { des }}(i, j)(i, j=1,2,3,4)$ on each element of the $4 \times 4$ output sensitivity function $\mathbf{S}_{o}(\mathrm{~s})=\mathbf{1}_{4}-\mathbf{T}_{\mathbf{w} \rightarrow \mathbf{z}}(\mathrm{s})$ where $\mathbf{T}_{\mathbf{w} \rightarrow \mathbf{z}}(\mathrm{s})$ is the closed-loop transfer between $\mathbf{w}$ and $\mathbf{z}$ as depicted in Figure 3:

$\gamma_{1}=\max _{i, j=1,2,3,4}\left\|\frac{\mathbf{S}_{o}(i, j)}{\mathbf{S}_{o, \text { des }}(i, j)}(\mathrm{s})\right\|_{\infty} \leq 1$,

with:

$\mathbf{S}_{o, d e s}(i, j)=\left\{\begin{array}{l}\frac{1.4 \mathrm{~s}}{\mathrm{~s}+\omega_{i}}, \text { if: } i=j, \\ 0.14, \text { otherwise. }\end{array}\right.$ and :

$\omega_{1}=0.2, \omega_{2}=0.3, \omega_{3}=\omega_{4}=1 \mathrm{rad} / \mathrm{s}$.

Such a multivariate template allows to prescribe low-frequency disturbance rejection and reference input tracking inside the bandwidth $\omega_{1}, . . \omega_{4}$ for the servo-loops on $V, V_{z}, \beta$ and $\phi$, respectively, while ensuring cross couplings to be lower than $14 \%$.

To avoid too high gains in the feed-forward path of the control law and so to reduce as much as possible the thrust magnitude in response to reference inputs, the decision variables must be tuned in order to limit the maximum of the 12 closed-loop propeller transfers $\mathbf{T}_{\mathbf{w} \rightarrow \widetilde{d}_{x, i}}$ :

$J_{1}=\max _{i=1, \cdots 12}\left\|\mathbf{T}_{\mathbf{w} \rightarrow \widetilde{d}_{x, i}}(\mathrm{~s})\right\|_{\infty}$.

This limitation can be done by minimizing the objective function $J_{1}$ under the constraint defined in (17), a methodology now common in the field of $H_{\infty}$ structured control design thanks to non-smooth optimization tools [4]. The idea here is to minimize the sizing parameters $\delta_{v}$ and $\omega_{p}$ using two additional objective functions:

$J_{2}=\delta_{v} \quad$ and $\quad J_{3}=\omega_{p}$.

In the previous work (see [20]), a sequential co-design procedure was retained to solve this multi-objective optimisation problem. It consisted in optimising for the first objective $\widehat{J}_{1}$, then adding as constraint a sub-optimal maximum gain $\bar{J}_{1} \leq \widehat{J}_{1}$ and optimizing for the next objective until the last objective is minimized. This methodology requires a manual adjustment of the trade-off between each objective functions and was justified to gain a better understanding of the problem.

The objective of this paper is to adapt the methodology to obtain a trade-off through the optimisation. This is achieved essentially by the addition of constraints translating actuator saturations and flight envelop requirements. 


\subsection{Towards a direct co-design}

Additional constraints are formulated based on flight envelop and flight handling quality constraints. For the aircraft to achieve a certain flight envelop in side slip using differential thrust, the motor power levels must be within the continuous power range. Additionally, to avoid disturbing the longitudinal dynamics, differential thrust must be antisymmetric. The motor saturation limits will hence depend on the trim level $d_{x_{i}, \text { trim }}$ and are introduced:

$\bar{d}_{x, i}=\min \left[1-\max \left(d_{x_{i}, \operatorname{trim}}\right), \min \left(d_{x_{i}, \operatorname{trim}}\right)\right]$,

$\underline{d}_{x, i}=\max \left(1-d_{x_{i}, \text { trim }}, d_{x_{i}, \text { trim }}\right)$.

The first saturation limit is $\bar{d}_{x, i}$. Beyond this point and up to $\underline{d}_{x, i}$, anti-symmetric differential thrust is not respected, resulting in perturbations on the longitudinal axis. The second saturation limit $\underline{d}_{x, i}$ represents the maximum yawing moment that can be generated by differential thrust.

The level of thrust during a manoeuvre for a pilot input signal $\beta_{r}(s)$ is given by $\tilde{d}_{x, i}(s)=\beta_{r}(s) \mathrm{T}_{\tilde{\beta}_{r} \rightarrow \tilde{d}_{x, i}}(s)$. Using the motor saturation limits, a constraint can be expressed to limit the gain of the transfer function $\mathrm{T}_{\tilde{\beta}_{r} \rightarrow \tilde{d}_{x, i}}$ :

$\bar{d}_{x, i} \geq\left|d_{x, i}(s)\right|=\left|\beta_{r}(s) \mathrm{T}_{\beta_{r} \rightarrow d_{x, i}}(s)\right|$.

Equation (22) must be enforced for steady states to ensure a certain achievable directional flight envelop. In this case using the final value theorem, equation (22) becomes:

$\bar{d}_{x, i} \geq \lim _{s \rightarrow 0}\left|d_{x, i}(s)\right|=\lim _{s \rightarrow 0}\left|s \beta_{r}(s) \mathrm{T}_{\beta_{r} \rightarrow d_{x, i}}(s)\right|$,

if $\beta_{r}(s)$ is a step of amplitude $\beta_{\text {req }}$, then:

$\bar{d}_{x, i} \geq \lim _{s \rightarrow 0}\left|d_{x, i}(s)\right|=\lim _{s \rightarrow 0}\left|s \frac{\beta_{\text {req }}}{s} \mathrm{~T}_{\beta_{r} \rightarrow d_{x, i}}(s)\right|$,

which can be reduced to:

$\left|\lim _{s \rightarrow 0} \mathrm{~T}_{\tilde{\beta}_{r} \rightarrow \tilde{d}_{x, i}}(s)\right| \leq \frac{\bar{d}_{x, i}}{\beta_{\text {req }}}$.

Constraint (23) requires that the absolute value of the steady state gain of the transfer function $\mathrm{T}_{\tilde{\beta}_{r} \rightarrow \tilde{d}_{x, i}}(s)$ should be lower than the first saturation limit $\bar{d}_{x, i}$ weighted by the required flight envelop side slip angle $\beta_{\text {req }}$.

During transient manoeuvres, it is possible that the imposed handling qualities require a large gain that can temporary saturate the motors. In this case, saturation is tolerable if it leads to slightly degraded handling qualities. However, it is desirable to avoid this scenario and the constraint (23) could be generalized to the full frequency range by constraining the maximum norm of equation (22) where $\beta_{r}(s)$ is a Dirac impulse with amplitude $\beta_{\text {req }}$ :

$\left\|\mathrm{T}_{\tilde{\beta}_{r} \rightarrow \tilde{d}_{x, i}}(s) \frac{\beta_{\text {req }}}{\bar{d}_{x, i}}\right\|_{\infty} \leq 1$.
Uncertainties remain on lateral closed loop transfers and more particularly on $\mathrm{T}_{\tilde{\beta}_{r} \rightarrow \tilde{d}_{x, i}}(s)$. Based on study [20], it is not clear which design variable influences most the thrust level required to achieve prescribed handling qualities and flight envelop. Additionally, it is unsure that equation (24) and equation (17) can be both satisfied, which would lead to a dead end during optimisation. To answer these questions and refine the constraints definition, a sensitivity analysis is conducted using the framework already available.

\subsection{Sensitivity analysis}

The idea for the sensitivity analysis is to optimize the system for minimum control gains (objective $J_{1}$, equation (18)) under handling qualities (17), and track the transfer function $\widehat{\mathrm{T}}_{\tilde{\beta}_{r} \rightarrow \tilde{d}_{x, i}}(s)$. After optimizing for $J_{1}, \widehat{\mathrm{T}}_{\tilde{\beta}_{r} \rightarrow \tilde{d}_{x, i}}(s)$ represents the minimum transfer function needed to achieve the desired handling qualities with the imposed set of vertical tail and motor bandwidth. Key quantities are then calculated from $\widehat{\mathrm{T}}_{\tilde{\beta}_{r} \rightarrow \tilde{d}_{x, i}}(s)$ : its steady state gain $C_{1}$ to measure the achievable flight envelop, its $H_{\infty}$ norm $C_{2}$ and corresponding frequency $C_{3}$ for transient manoeuvres.

$$
\begin{aligned}
C_{1} & =\frac{\lim _{s \rightarrow 0} \widehat{\mathrm{T}}_{\tilde{\beta}_{r} \rightarrow \tilde{d}_{x, i}}(s)}{\left|\lim _{s \rightarrow 0} \widehat{\mathrm{T}}_{\tilde{\beta}_{r} \rightarrow \tilde{d}_{x, i}}(s)\right|} \max _{i=1, \cdots 12}\left|\lim _{s \rightarrow 0} \widehat{\mathrm{T}}_{\tilde{\beta}_{r} \rightarrow \tilde{d}_{x, i}}(s)\right|, \\
C_{2} & =\max _{i=1, \cdots 12}\left\|\widehat{\mathrm{T}}_{\tilde{\beta}_{r} \rightarrow \tilde{d}_{x, i}}(s)\right\|_{\infty}, \\
C_{3} & =\omega_{H_{\infty}},
\end{aligned}
$$

such that:

$$
\max _{i=1, \cdots 12}\left|\widehat{\mathrm{T}}_{\tilde{\beta}_{r} \rightarrow \tilde{d}_{x, i}}\left(j \omega_{H_{\infty}}\right)\right|=C_{2} \text {. }
$$

The set of parameters that are varied in the analysis is $\delta_{\mathbf{p}}=\left[\delta_{v}, \omega_{p}, \omega_{3}, \bar{q}\right]$, respectively, the vertical tail surface area, motor cut-off frequency, handling quality template cutoff frequency for the side slip and the dynamic pressure. This last parameter is varied by changing the flight condition.

The standard configuration, as starting point for this sensitivity analysis is detailed in Table 2.

A drawback of non-smooth optimization tool is the possibility to end in a local minimum. This is normally avoided by running multiple optimization with random initial solution. To avoid too high computational cost, an intermediate solution consisted in initializing the control gains by eigenstructure assignment (see [7] and [20]). This initialization method allowed to find solutions as good as with multiple random start but also ensures reproducibility of the results.

The evolution of $C_{1}, C_{2}$ and $C_{3}$ with variation of parameters $\delta_{\mathbf{p}}$ is shown in Figure 5 to Figure 9. 
Table 2: Standard configuration for sensitivity analysis.

\begin{tabular}{l|c}
\multicolumn{2}{c}{ Optimisation settings } \\
\hline Constraints & $\gamma_{1} \leq 1$ \\
Objective function & $J_{1}$ \\
\hline Parameters & Values \\
\hline$\delta_{v}$ & 1 \\
$\omega_{p}$ & $20(\mathrm{rad} / \mathrm{s})$ \\
$\omega_{3}$ & $1(\mathrm{rad} / \mathrm{s})$ \\
$\bar{q}$ & $0.5 \rho_{0}\left(1.3 V_{s r}\right)^{2}$
\end{tabular}

\subsubsection{Variation of Vertical tail surface area and motor bandwidth}

The influence of $\delta_{v}$ and $\omega_{p}$ on $C_{1}, C_{2}$ and $C_{3}$ is represented in surface plots in Figure 5, Figure 6 and Figure 7.

The analysis of Figure 5 reveals that the vertical tail (VT) size has a major influence on the minimum steady state gain, while the motor bandwidth has negligible effects on it. This is a reasonable result, as a large vertical tail brings a natural stability to the aircraft, or inversely, an important aircraft instability requires a larger control effort to maintain a certain side slip. It is worth to mention that the evolution of the steady state gain as a function of $\delta_{v}$ is close to linear. This effect was anticipated in [22] where it was shown that a variation of surface area at constant aspect ratio translates as a linear variation of lateral coefficients.

From Figure 6, the motor bandwidth has the most influence on the $H_{\infty}$ norm which shows an important increase for motor bandwidths lower than $10 \mathrm{rad} / \mathrm{s}$. The vertical tail size has a small influence. The control effort is reduced for larger surface area but its contribution is much smaller than the motor bandwidth. Figure 7, confirms that the maximum control effort is necessary for transient manoeuvres as $C_{3}$ does not go below $4 \mathrm{rad} / \mathrm{s}$ and increases with increasing motor bandwidth.

\subsubsection{Variation of side slip handling quality template cut-off frequency}

Increasing $\omega_{3}$ translates as a faster aircraft answer when an input is issued by the pilot. Consequently and as shown by Figure $8 \mathrm{~b}, C_{2}$ increases with increasing $\omega_{3}$. Doubling the flight handling requirement from $\omega_{3}=0.6 \mathrm{rad} / \mathrm{s}$ to $\omega_{3}=$ $1.2 \mathrm{rad} / \mathrm{s}$, necessitates to quadruple $C_{2}$, showing the sensitivity of this parameter. $C_{1}$ is shown to be loosely dependent on $\omega_{3}$ (see Figure 8a) while $C_{3}$ shows a increasing tendency with increasing $\omega_{3}$ (see Figure 8c). When comparing the value of $C_{1}$ and $C_{2}$ in Figures $8 \mathrm{a}$ and $8 \mathrm{~b}$, one can observe that in standard configuration $C_{2}$ is three times more important than $C_{1}$.

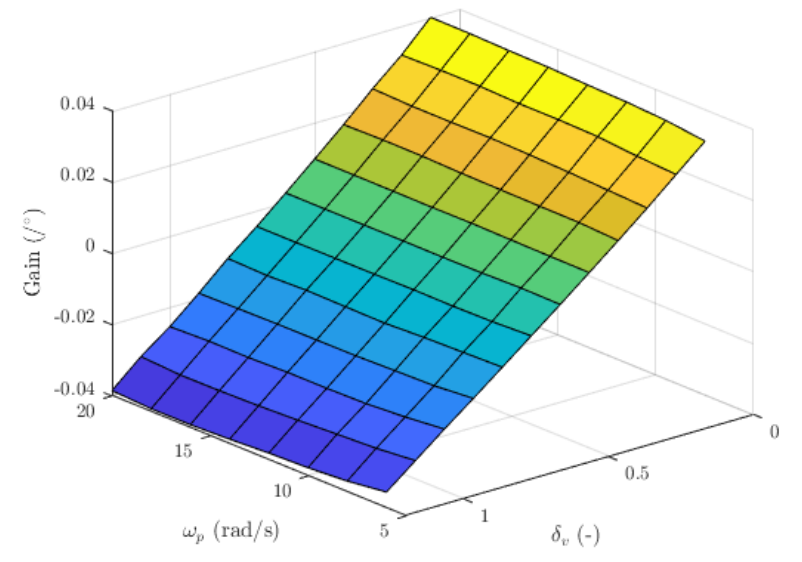

Fig. 5: Evolution of the steady state gain $C_{1}$, with motor bandwidth, $\omega_{p}$ and VT size $\delta_{v}$.

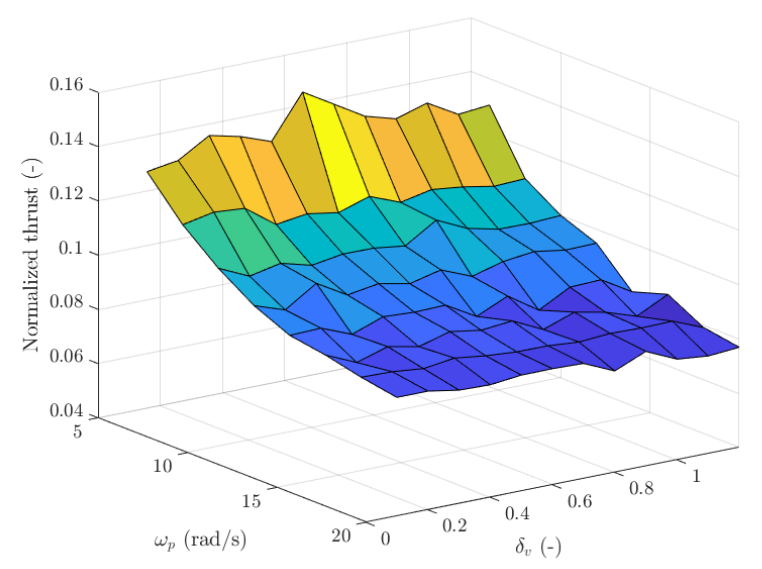

Fig. 6: Evolution of the $H_{\infty}$ norm $C_{2}$, with motor bandwidth, $\omega_{p}$ and VT size, $\delta_{v}$.

\subsubsection{Variation of dynamic pressure}

Four flight conditions are studied and allow an estimation of the variation of dynamic pressure:

- $1.3 V_{s r}$, steady flight at air velocity 1.3 times higher than the stall speed ${ }^{1} V_{s r}$ (see Table 1).

- $1.3 V_{s r}$ FL100, same as the previous but at the flight level FL100, altitude of 10, 000ft or $3048 \mathrm{~m}$.

- $90 \mathrm{~m} / \mathrm{s}$, a velocity corresponding to best climb rate.

- $90 \mathrm{~m} / \mathrm{s}$ FL100, same as previous at the flight level FL100, altitude of $10,000 \mathrm{ft}$ or $3048 \mathrm{~m}$.

Figure $9 \mathrm{a}$ and $9 \mathrm{~b}$ show an important sensitivity of $C_{1}$ and $C_{2}$ to the airspeed velocity, as expected from the thrust model

\footnotetext{
1 All indicated airspeed are true airspeed
} 


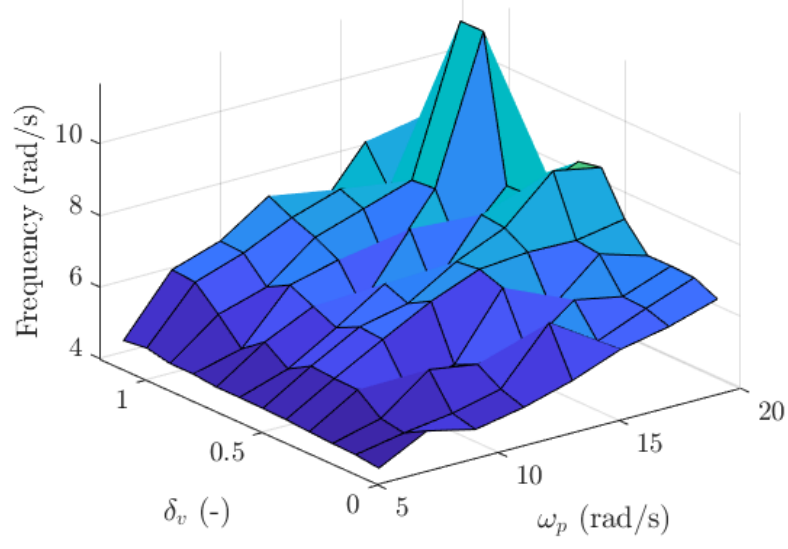

Fig. 7: Evolution of the $H_{\infty}$ frequency $C_{3}$, with motor bandwidth, $\omega_{p}$ and VT size, $\delta_{v}$.

(equation (3)). The same figures indicate that for a constant airspeed, the control effort in transient steady manoeuvres is more important for lower air density.

Figure 9c suggests a slower dynamic at higher altitudes for a constant airspeed.

\subsubsection{Interpretation}

The most surprising result is the fact that the vertical tail surface area has a low influence on $C_{2}$, with respect to motor bandwidth. The primary focus for limiting the transient gain should be put on motor bandwidth rather than natural stability. This can be related to the fact that a yaw damper is often added to transport aircraft regardless of the vertical tail size [1].

The large difference between $C_{1}$ and $C_{2}$ can provide an explanation for this result. During steady state, motors only have to counter the aircraft natural stability mainly given by the vertical tail. When $C_{2}$ is much larger than $C_{1}$, the vertical tail surface area represents only a small added effort in transient manoeuvres with respect to inertia. The sensitivity of $C_{2}$ with respect to $\delta_{v}$ can increase as $C_{2}$ approaches $C_{1}$ values. At this point, efforts due to inertia and those due to aerodynamics should be of similar magnitude.

When decreasing the motor bandwidth, the fact that $C_{2}$ increases rapidly while $C_{3}$ remains higher than $4 \mathrm{rad} / \mathrm{s}$ suggests that this is a particularly important frequency for the actuator to cover.

Reaching a prescribed directional flight envelop seems easily feasible by adjusting the vertical tail size. The motor bandwidth should be kept larger than $\omega_{p}>10 \mathrm{rad} / \mathrm{s}$ to avoid large $C_{2}$ values and this value is compatible with electric motors which have reaction times of the order of $10^{-1} \mathrm{~s}$ [14].

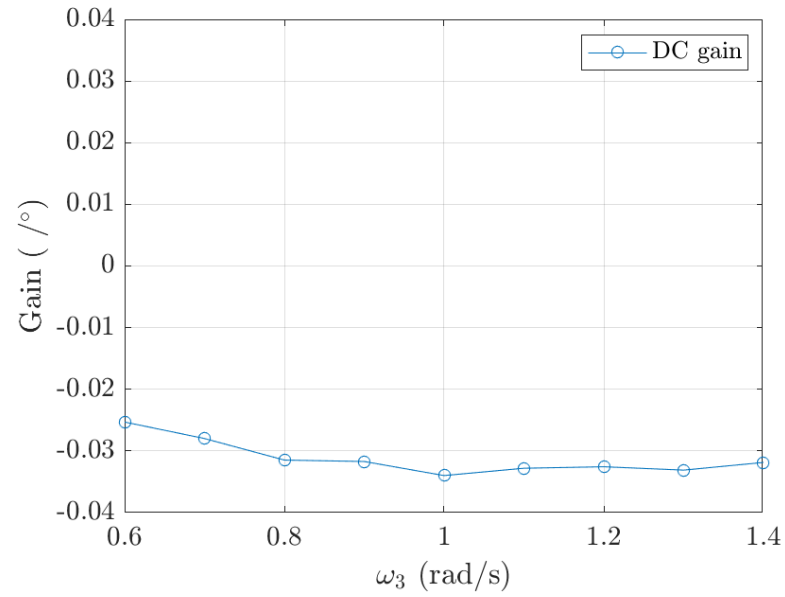

(a) Steady state gain $C_{1}$ evolution with $\omega_{3}$

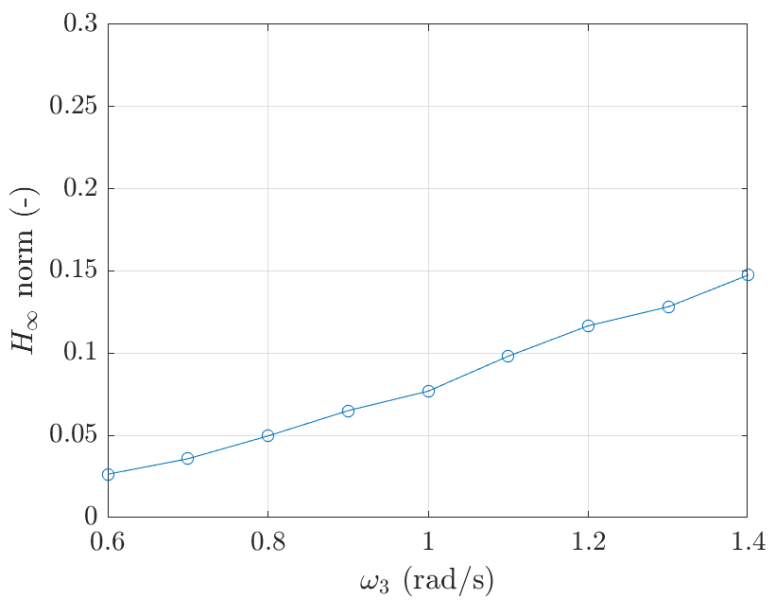

(b) $H_{\infty}$ gain $C_{2}$ evolution with $\omega_{3}$

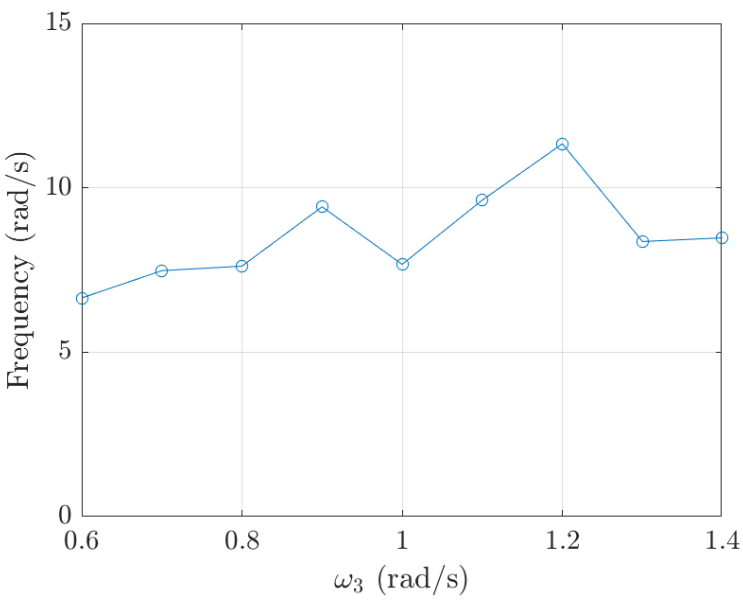

(c) $H_{\infty}$ frequency $C_{3}$ evolution with $\omega_{3}$

Fig. 8: Sensitivity analysis in function of the side slip cut-off frequency $\omega_{3}$ 


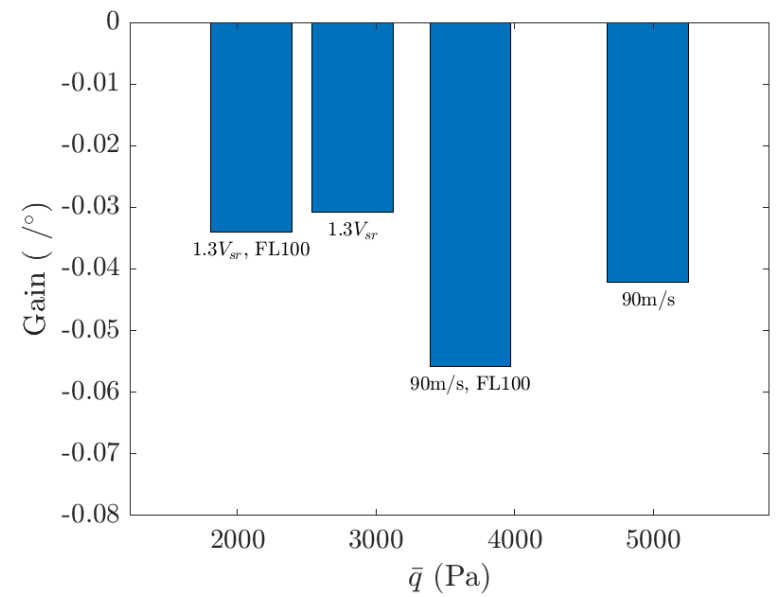

(a) Steady state gain $C_{1}$ evolution with $\bar{q}$

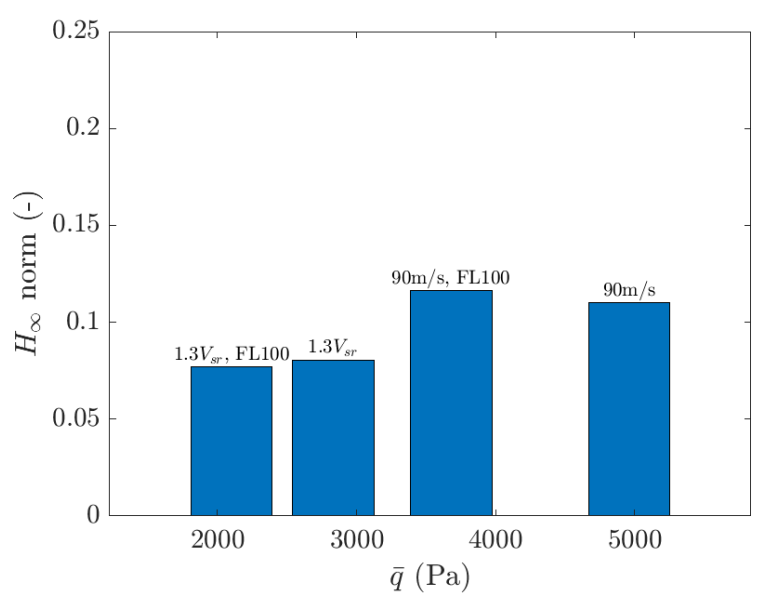

(b) $H_{\infty}$ gain $C_{2}$ evolution with $\bar{q}$

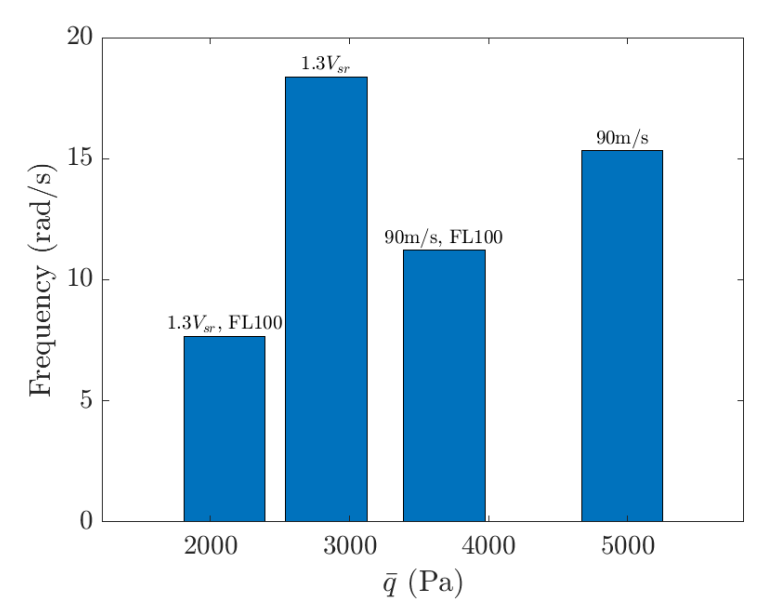

(c) $H_{\infty}$ frequency $C_{3}$ evolution with $\bar{q}$

Fig. 9: Sensitivity analysis in function of the dynamic pressure $\bar{q}$

\subsection{Consequences for direct co-design}

Recalling the constraints (23) and (24) of section 3.1:

$$
\begin{aligned}
& \left|\lim _{s \rightarrow 0} \mathrm{~T}_{\tilde{\beta}_{r} \rightarrow \tilde{d}_{x, i}}(s)\right| \leq \frac{\bar{d}_{x, i}}{\beta_{\text {req }}}, \\
& \left\|\mathrm{T}_{\tilde{\beta}_{r} \rightarrow \tilde{d}_{x, i}}(s) \frac{\beta_{\text {req }}}{\bar{d}_{x, i}}\right\|_{\infty} \leq 1,
\end{aligned}
$$

it is now possible to evaluate if these constraints are realizable for a required side slip $\beta_{\text {req }}=15^{\circ}$. This value is chosen based on certification specification paragraph CS25.147. The steady state gain can be easily limited to reach a prescribed directional flight envelop by adjusting the vertical tail size. This constraint allows a naturally unstable aircraft where actuator saturation is potentially catastrophic and must be avoided. For steady states, equation (23) is sufficient to avoid motor saturation.

For transient manoeuvres, it is necessary to evaluate the factor $\frac{\bar{d}_{x, i}}{\beta_{\text {req }}}$ and compare it with Fig $8 \mathrm{~b}$ and Fig $8 \mathrm{~b}$. At the flight condition corresponding to $1.3 V_{\mathrm{sr}}$ FL0, the trim power level is $d_{x, i}=0.357$, then $\frac{\bar{d}_{x, i}}{\beta_{\text {req }}}=0.024 /{ }^{\circ}$. To satisfy constraint (24) and avoid saturating motors during transient manoeuvres, one must have: $\left\|\mathrm{T}_{\tilde{\beta}_{r} \rightarrow \tilde{d}_{x, i}}(s)\right\|_{\infty} \leq \frac{\bar{d}_{x, i}}{\beta_{\text {req }}}$. Based on Fig $8 \mathrm{~b}$ and Fig $8 \mathrm{~b}$, this is not possible.

Using the second saturation limit $\underline{d}_{x, i}$ in constraint (24) for transient manoeuvres allows more margin with the corresponding factor $\frac{d_{x, i}}{\beta_{\text {req }}}=0.043$. This allows the handling quality template cut-off frequency to be set at $\omega_{3}=0.7 \mathrm{rad} / \mathrm{s}$. Since the longitudinal $V-\gamma$ mode is much slower than the directional mode, the perturbation associated with the utilization of the second saturation limit should have a low impact on the longitudinal axis and may be considered for dynamic manoeuvres.

Constraints are hence defined with two levels of severity. The most severe saturation limit is used for static equilibrium, the less severe is used for dynamic manoeuvres.

\subsubsection{Actuator frequency template}

Both steady state and dynamic gain limitations can be obtained by imposing a frequency-domain template between the lateral reference inputs and motor control signals $\mathrm{T}\left(s, \bar{d}_{x, i}, \underline{d}_{x, i}\right)$, of the form given by equation (28) and illustrated in Figure 10.

$\mathrm{T}\left(s, \bar{d}_{x, i}, \underline{d}_{x, i}\right)=\frac{\left(s+\omega_{a}\right)^{2}}{\left(a s+\omega_{b}\right)^{2}}$,

with:

$$
\begin{aligned}
\lim _{s \rightarrow 0} \mathrm{~T}\left(s, \bar{d}_{x, i}, \underline{d}_{x, i}\right) & =\frac{1}{a^{2}}=\frac{\bar{d}_{x_{i}}}{\beta_{\text {req }}}, \\
\lim _{s \rightarrow \infty} \mathrm{T}\left(s, \bar{d}_{x, i}, \underline{d}_{x, i}\right) & =\left(\frac{\omega_{a}}{\omega_{b}}\right)^{2}=\frac{\underline{d}_{x_{i}}}{\beta_{\text {req }}} .
\end{aligned}
$$




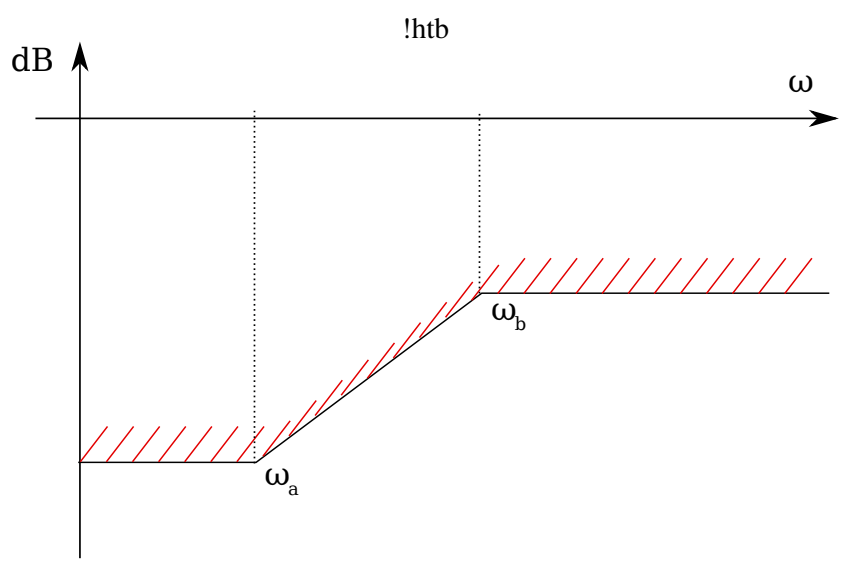

Fig. 10: $\mathrm{T}\left(s, \bar{d}_{x, i}, \underline{d}_{x, i}\right)$ template definition.

The required side slip $\beta_{\text {req }}=15^{\circ}$ is selected based on regulation specification paragraph CS25.147. As for $\omega_{b}$, it has to be chosen based on Fig 7 and Fig 8c. A value of $\omega_{b}=2$ $\mathrm{rad} / \mathrm{s}$ can be reasonably chosen.

Remains the definition of an adequate gain limitation for longitudinal reference inputs. From the previous study [20], it was shown that a constant gain is sufficient. Let $\varepsilon_{V}=0.15$ be the maximum longitudinal gain, the longitudinal constraints writes:

$\left\|\frac{1}{\varepsilon_{V, \gamma}} \mathbf{T}_{\widetilde{V}_{r}, \widetilde{V}_{z_{r}} \rightarrow \tilde{d}_{x, i}}(s)\right\|_{\infty} \leq 1$.

All control gains being constrained, the objective function is defined in terms of design variables only:

$J=\delta_{v}+\frac{\omega_{p}}{\omega_{p, 0}}$

representing the sum of normalized $J_{2}$ and $J_{3}$, where $\omega_{p_{0}}=$ $20 \mathrm{rad} / \mathrm{s}$. All constraints and the objective function are resumed in Table 4.

\subsubsection{Initialization}

Structured $H_{\infty}$ control design being non-convex, the solution depends on the initial solution provided. In order to guide the solver toward a feasible solution, the decision variables are initialized in the following way:

- the initial A/C configuration is defined by $\delta_{v}=1.0$ and the avionics are assumed perfect,

- the allocation matrix $\mathbf{A}$ is initialized with: $\mathbf{L}_{L}=\mathbf{1}_{6}^{T}$, $\mathbf{L}_{D}=\mathbf{1}_{6}^{T}$,

- then, $K_{\alpha}$ and $K_{q}$ are designed to assign the short-period mode to the roots of $\left(\frac{\mathrm{s}}{1.2}\right)^{2}+1.4 \frac{\mathrm{s}}{1.2}+1$ on the 2 -nd order short-term longitudinal model (also called $(\alpha-q)$ model),
- $\mathbf{K}_{L}$ is designed to assign the eigenstructure of the longitudinal long-term model (also called $(V-\gamma)$ model), i.e. place the 2 corresponding eigenvalues to -0.2 and -0.3 while decoupling the associated eigenvectors from $\gamma$ or $V$ respectively. See more details in [10]. $\mathbf{H}_{L}$ is then computed to have an identity DC-gain between $\left[\widetilde{V}_{r}, \widetilde{\gamma}_{r}\right]^{T}$ and $[\widetilde{V}, \widetilde{\gamma}]^{T}$ on this $(V-\gamma)$ model,

- $\mathbf{K}_{D}$ is designed to assign the 4 eigenvalues of the lateral model to $-1 \pm j$ for the Dutch Roll, -1 for the Spiral, -20 for the Roll Subsidence, and the associated eigenvectors decoupled from $\phi$, resp. $\beta$. See more details in [10]. $\mathbf{H}_{D}$ is then computed to have an identity DC-gain between $\left[\widetilde{\beta}_{r}, \widetilde{\phi}_{r}\right]^{T}$ and $[\widetilde{\beta}, \widetilde{\phi}]^{T}$,

- Finally, the actuators are initialized with $\omega_{p}=\omega_{e}=$ $\omega_{a}=20 \mathrm{rad} / \mathrm{s}$.

\subsection{Direct Co-design Results}

The results of the co-design are available in Table 3.

Table 3: Co-design results

\begin{tabular}{c|c|c}
$\max _{i=1,2,3} \gamma_{i}$ & $\delta_{v}$ & $\omega_{p}(\mathrm{rad} / \mathrm{s})$ \\
\hline 0.9997 & 0.4 & 8.0
\end{tabular}

A trade off has been found between vertical tail surface area and motor bandwidth. The motor bandwidth has been reduced to $8.0 \mathrm{rad} / \mathrm{s}$, corresponding to a rather high $C_{2}$ value. A vertical tail surface area of 0.4 is found, corresponding to an unstable aircraft in open loop.

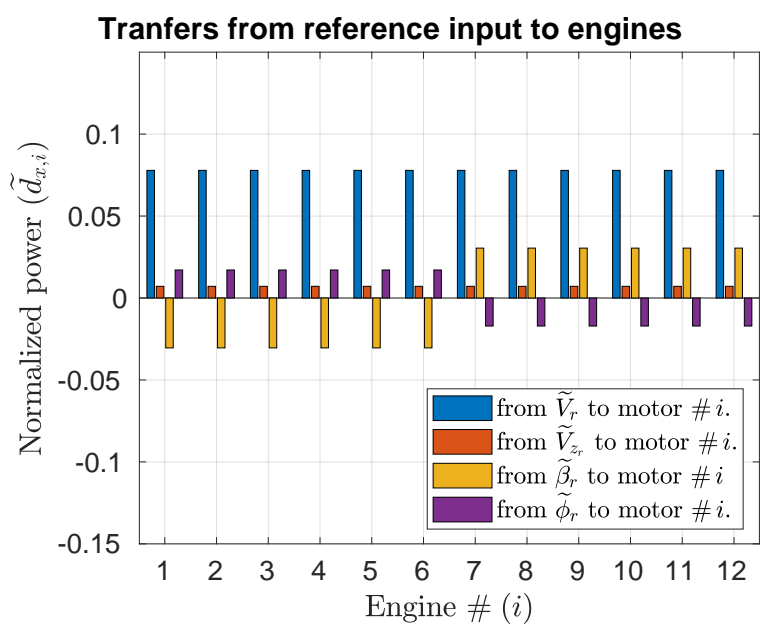

Fig. 11: Transfer from reference input to motor control inputs. 
Table 4: Direct co-design formulation

\begin{tabular}{|c|c|c|}
\hline \multicolumn{3}{|l|}{ Co-design } \\
\hline Variables & $\mathcal{K}_{2}=\left[\mathbf{H}_{L}, \mathbf{H}_{D}, \mathbf{K}_{L}, \mathbf{K}_{D}, \mathbf{L}_{L}, \mathbf{L}_{D}, K_{\alpha}, K_{q}, \delta_{v}, \frac{\omega_{p}}{\omega p_{0}}\right]$ & $\widehat{\mathcal{K}}_{2}=\arg \min _{\mathcal{K}_{2}} J_{\text {co }}$ \\
\hline Objective & $J_{\mathrm{co}}=\delta_{v}+\frac{\omega_{p}}{\omega_{p}}$ & Such that: \\
\hline \multirow{4}{*}{ Constraints } & Flight handling qualities & $\left\|\frac{\mathbf{S}_{0}(i, j)}{\mathbf{S}_{0, d e s}(i, j)}\right\|_{\infty} \leq \gamma_{1}$ \\
\hline & Directional flight envelop & $\mid \mathbf{T}_{\tilde{\beta}_{r}, \tilde{\phi}_{r} \rightarrow \tilde{d}_{x, i}}(s)$ \\
\hline & Motor saturation & $\left\|\mathrm{T}\left(s, \bar{d}_{x, i}, \underline{d}_{x, i}\right)\right\|_{\infty}$ \\
\hline & Longitudinal gain limitation & $\left\|\frac{1}{\varepsilon_{V, \gamma}} \mathbf{T}_{\widetilde{V}_{r}, \widetilde{V}_{z_{r}} \rightarrow \tilde{d}_{x, i}}(s)\right\|_{\infty} \leq \gamma_{3}$ \\
\hline
\end{tabular}

The allocation from reference input to motor control input $\mathbf{A}_{12 \times 2}\left[\begin{array}{cc}\mathbf{H}_{L}(2,:) & \mathbf{0}_{1 \times 2} \\ \mathbf{0}_{1 \times 2} & \mathbf{H}_{D}(2,:)\end{array}\right] \mathbf{S}$ is represented in Fig 11 . The maximum directional control power is sought, as all motors are uniformly used for lateral inputs.

The fact that a constant allocation is found for all motors shows that for this particular aircraft, considering each motor as an individually actuator is not necessary in normal operation. Regrouping motors in clusters as done in [9] would reduce complexity without loss of performances.
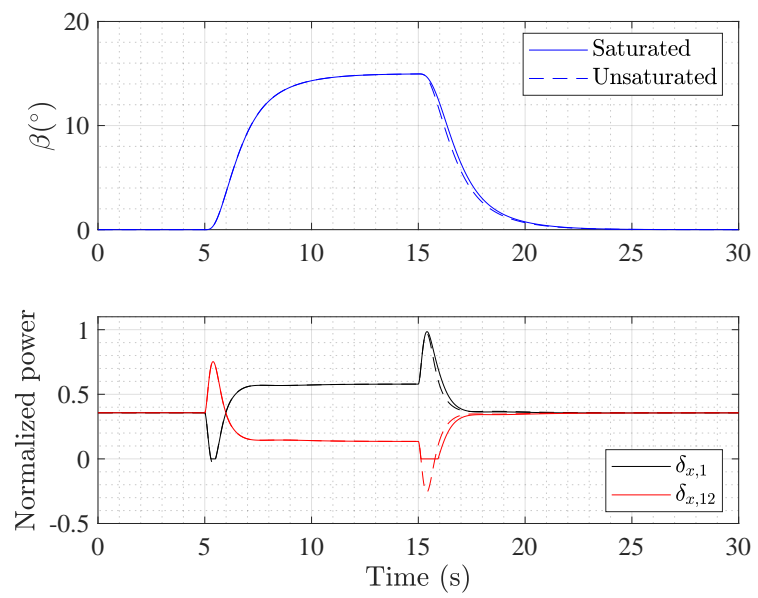

Fig. 12: Answer to a $\beta_{r}=15^{\circ}$ doublet input. Dashed line, unsaturated actuators, continuous line, saturated actuator.

All constraints are satisfied at least in the linear domain. To estimate the performance degradation due to motor saturations, a time simulation of a $\beta_{r}=15^{\circ}$ step input followed by a return to neutral is presented in Figure 12. As the resulting design is unstable, the most difficult manoeuvre is to go from $\beta=15^{\circ}$ back to $\beta=0^{\circ}$ due to the proximity of the saturation limits. The full lateral and longitudinal states are available in Fig 13 and in Fig 14, respectively.

Figure 12 shows that as expected the closest saturation limit is hit, resulting in a small performance degradation.
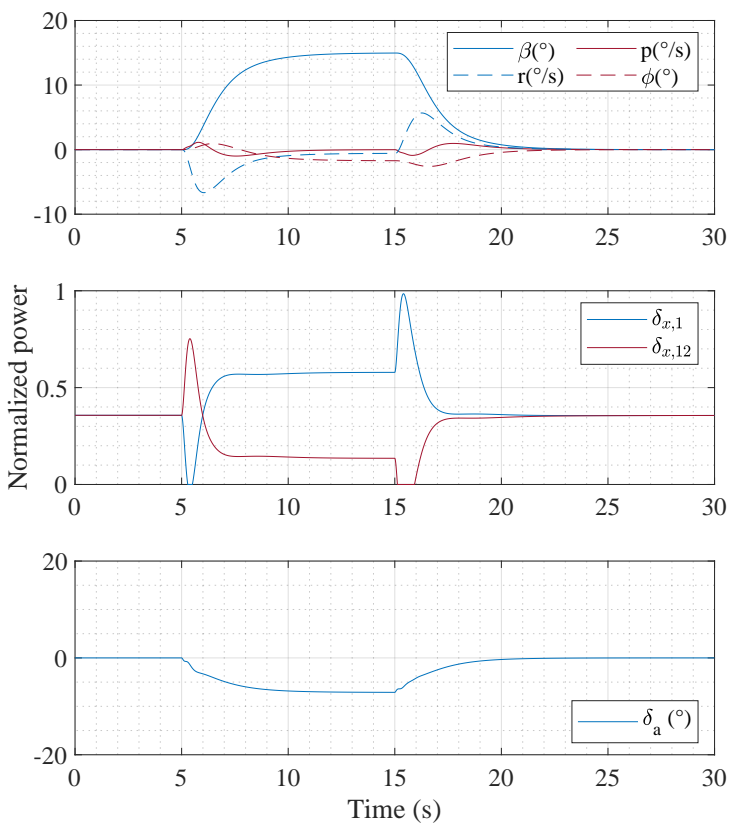

Fig. 13: Lateral states answer to a $\beta_{r}=15^{\circ}$ doublet input.

The induced perturbation on the longitudinal states does not exceed $V=0.14 \mathrm{~m} / \mathrm{s}$ (see Fig 14).

The analysis of the closed loop poles of the aircraft before and after co-design is given in Fig 15 and confirms that the aircraft is rendered stable. A couple of badly damped complex conjugate poles are not fixed during the initialization. These arise from the combination of the ailerons actuator dynamic and the roll motion. After the co-design, these poles are better damped $(\xi \approx 0.5)$ but since there is no explicit constraint on the poles damping these may be poorly damped after the co-design. In this case, it was not found necessary but a constraint requiring all gains to be sufficiently damped would solve this problem.

To obtain a feasible solution, the directional handling qualities had to be slowed down at $\omega_{3}=0.7 \mathrm{rad} / \mathrm{s}$, translating in a time constant of $1.43 \mathrm{~s}$. This dynamic may be considered too slow and can constitute an argument against the 

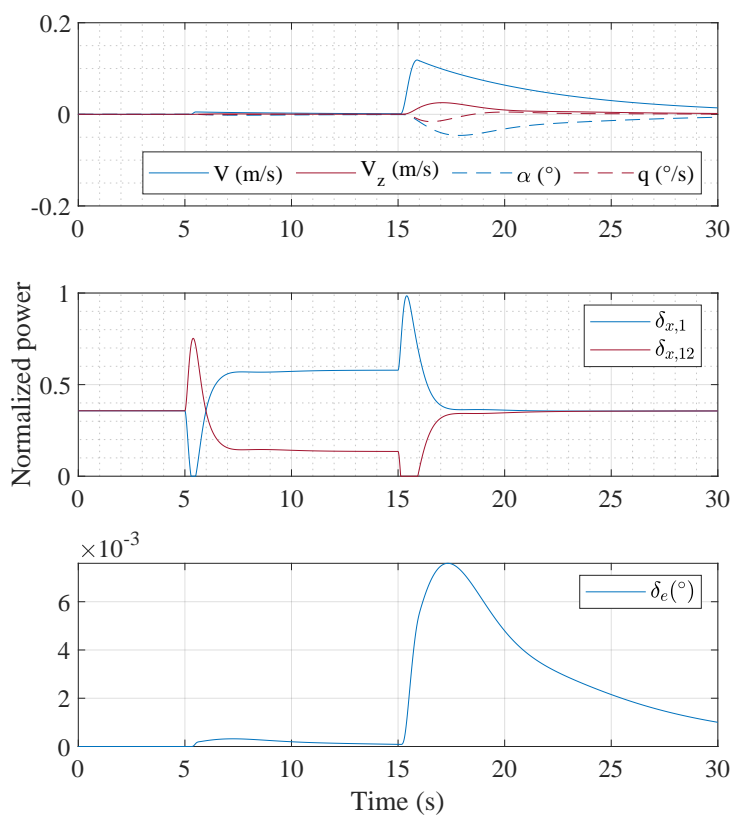

Fig. 14: Longitudinal states answer to a $\beta_{r}=15^{\circ}$ doublet input.

complete removal of the rudder on aircraft with high inertia like the ATR72. The directional dynamics can be increased by re-introducing a rudder. Then an allocation between rudder and differential thrust could logically be a split of the input signal between high frequency components for the rudder and near steady components for differential thrust. The inertia being a strong limiter to the reduction of the vertical tail, the installation of the distributed propulsive chain on the aircraft should be made with the idea to minimize the inertia terms $I_{x}$ and $I_{z}$.

This co-design was performed at one flight point. Exploration at every expected flight condition must now be considered to find the most demanding flight conditions for the vertical tail and motor bandwidth in nominal flight operation. In parallel, the design of the vertical tail cannot be definitive without studying the case of critical motor failure. The same framework can incorporate a flight scenario with asymmetric thrust. The large vertical tail reduction obtained in a normal flight condition leaves enough margin to find a trade-off with still consequent vertical tail reduction during degraded flight conditions.

The resulting design being unstable, the system modelling can be considered too simplistic, or the vertical tail reduction too optimistic for the following reasons:

- absence of delays in the avionics,

- no rate saturation,

- absence of atmospheric perturbation,

- no aero-propulsive interaction effects.

\section{Conclusion}

A co-design framework to dimension at the same time vertical tail surface area, motor bandwidth and compute the control laws has been presented for an aircraft equipped with active distributed electric propulsion. Handling qualities and flight envelop requirements have been included in the codesign constraints so as to explore only feasible solutions with the help of a sensitivity analysis. It was found that motor bandwidth has the most impact on the control effort necessary to satisfy handling quality requirements, such that the focus should be put on sufficient motor bandwidth rather than aircraft natural stability. Motor saturation cannot be avoided with the required handling qualities. As a consequence, the entire replacement of the rudder by differential thrust on an aircraft such as the ATR72 is not recommended. Nevertheless, a solution was found with a vertical tail reduced by $60 \%$ and an motor bandwidth of $8.0 \mathrm{rad} / \mathrm{s}$ with very limited performance degradation. The replacement of vertical tail by active differential thrust can remain advantageous for aircraft having a lower inertia.

\section{Conflict of interest}

The authors declare that they have no conflict of interest.

\section{References}

1. Abzug, M., Larrabee, E.: Airplane Stability and Control: A History of the Technologies that Made Aviation Possible. Cambridge Aerospace Series. Cambridge University Press (2005). URL https://books.google.fr/books?id=wpYDYLuOix4C

2. Federal Aviation Administration: Type certificate data sheet no. a53eu (2015)

3. Ameyugo, G., Taylor, M., Singh, R.: Distributed propulsion feasability studies. ICAS06 (2006)

4. Apkarian, P.: Tuning Controllers Against Multiple Design Requirements. In: System Theory, Control and Computing (ICSTCC), 2012 16th International Conference on, pp. 1-6. IEEE (2012)

5. Bushnell, D.M.: Aircraft drag reduction - a review. Journal of Aerospace Engineering 217(1) (2003)

6. Ciliberti, D., Vecchia, P.D., Nicolosi, F., Marco, A.D.: Aircraft directional stability and vertical tail design: A review of semiempirical methods. Progress in Aerospace Sciences 95, 140 - 172 (2017). DOI https://doi.org/10.1016/j.paerosci.2017.11.001

7. Denieul, Y.: Preliminary Design of Control Surfaces and Laws for Unconventional Aircraft Configurations. Theses, Institut Supérieur de l'Aéronautique et de l'Espace (ISAE) (2016). URL https://hal.archives-ouvertes.fr/tel-01482103

8. Diederich, F.W.: A planform parameter for correletating certain aerodynamic characteristics of swept wing (NACA-TN-2335) (1951)

9. Dilinger, E., Döll, C., Liaboeuf, R., Toussaint, C., Hermetz, J., Verbeke, C., Ridel, M.: Handling qualities of ONERA's small business concept plane with distributed electric propulsion. ICAS18 Bello Horizonte, Brazil (2018) 

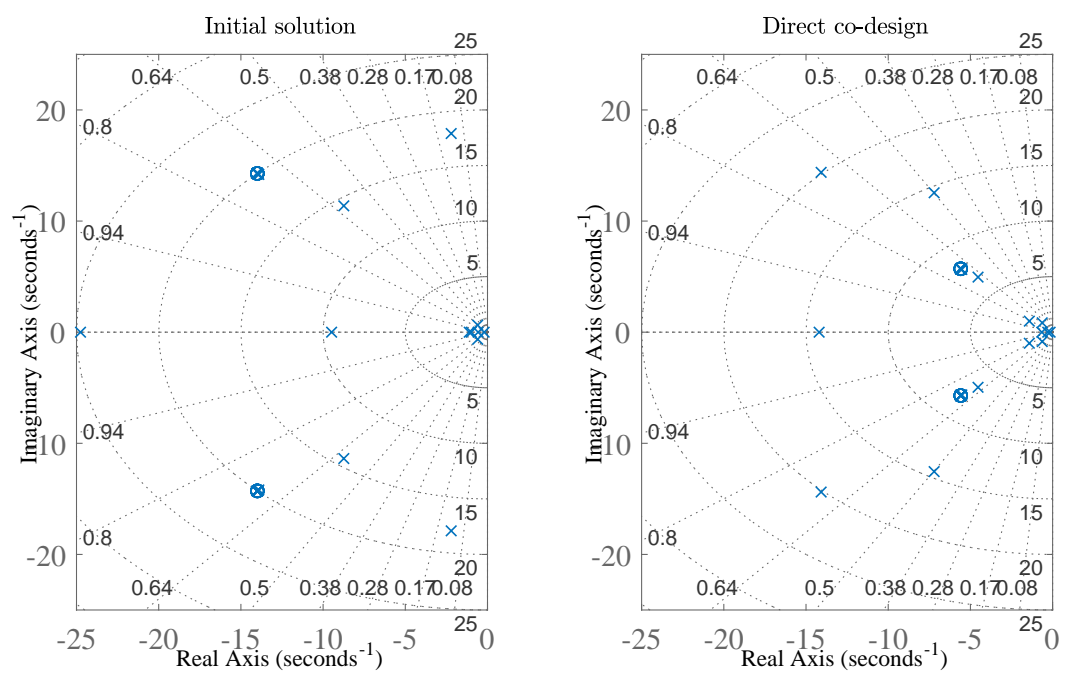

Fig. 15: Map of the poles before and after the co-design.

10. Döll, C., Magni, J., Le Gorrec, Y.: A modal multi-modal approach. In: J. Magni, S. Bennani, J. Terlouw (eds.) Robust flight control - A design challenge, vol. 224, 1st edn., chap. 19, pp. 258-277 (1997)

11. Etkin, B.: Dynamics of Atmospheric Flight. Dover Books on Aeronautical Engineering. Dover Publications (2012). URL https://books.google.fr/books?id=T_2X_YWTc88C

12. Felder, J.L., Kim, H.D., Brown, G.V.: Turboelectric distributed propulsion engine cycle analysis for hybrid wing body aircraft. $47^{\text {th }}$ AIAA Aerospace Sciences Meeting (1132) (2009). DOI $10.2514 / 6.2009-1132$

13. Gloudemans, J.R., Davis, J.D., Gelhausen, P.A.: A rapid geometry modeler for conceptual aircraft. AIAA, Aerospace Sciences Meeting and Exhibit, 34th, Reno, NV, Jan. 15-18, 1996 (1996)

14. Grellet, G., Clerc, G.: Actionneurs électriques: principes, modèles, commande. Collection Électrotechnique. Eyrolles (1996). URL https://books.google.fr/books?id=2dftPAAACAAJ

15. Hermetz, J., Ridel, M., Döll, C.: Distributed Electric Propulsion for small business aircraft - A concept-plane for keytechnologies investigations. In: Proc. 30th ICAS Congress. Daejeon, South Korea (2016)

16. Jackson, P.: Jane's all the world's aircraft: Development and production : 2014-2015 (2014). URL https://books.google.fr/books?id=bzhMzQEACAAJ

17. Ko, A., Schetz, J.A., Mason, W.H.: Assessment of the potential advantages of distributed propulsion for aircraft. ISABE-20031094 (2003)

18. Kraft, D.: A software package for sequential quadratic programming. No. DFVLR-FB 88-28 in Forschungsbericht / Deutsche Forschungs- und Versuchsanstalt für Luft- und Raumfahrt. Wissenschaftl. Berichtswesen d. DFVLR (1988)

19. Lan, C.E., Guan, M.: Flight dynamic analysis of a turboprop transport airplane in icing accident. AIAA Atmospheric Flight Mechanics Conference and Exhibit (AIAA 2005-5922) (2005)

20. Nguyen-Van, E., Alazard, D., D C.: Co-design of aircraft vertical tail and control laws using distributed electric propulsion. IFAC-PapersOnLine 52(12), 514 - 519 (2019). DOI https://doi.org/10.1016/j.ifacol.2019.11.295. 21st IFAC Symposium on Automatic Control in Aerospace ACA 2019

21. Nguyen-Van, E., Alazard, D., Pastor, P., Döll, C.: Reduction of vertical tail using differential thrust: Influence on flight control and certification. AEGATS'18, Toulouse, France (2018)
22. Nguyen-Van, E., Alazard, D., Pastor, P., Döll, C.: Towards an aircraft with reduced lateral static stability using electric differential thurst. AIAA Aviation Forum (AIAA2018-3209) (2018)

23. Nicolosi, F., Ciliberti, D., Della Vecchia, P., Corcione, S., Cusati, V.: A comprehensive review of vertical tail design. Aircraft Engineering and Aerospace Technology 89(4), 547 - 557 (2017)

24. Roos, C., Hardier, G., Biannic, J.: Polynomial and rational approximation with the apricot library of the smac toolbox. In: 2014 IEEE Conference on Control Applications (CCA), pp. 1473-1478 (2014)

25. Sachs, G.: Flight performance issues of electric aircraft. AIAA Atmospheric Flight Mechanics Conference (4727) (2012)

26. Schmollgruber, P., Atinault, O., Cafarelli, I., Döll, C., François, C., Hermetz, J., Liaboeuf, R., Paluch, B., Ridel, M.: Multidisciplinary Exploration of DRAGON: an ONERA Hybrid Electric Distributed Propulsion Concept. AIAA SciTech Forum (AIAA2019-1585) (2019). DOI 10.2514/6.2019-1585

27. Veldhuis, L.: Review of propeller wing aerodynamic interference. ICAS (2004) 